\title{
The American Journal of Sports Medicine
}

http://ajs.sagepub.com/

Characterized Chondrocyte Implantation Results in Better Structural Repair When Treating Symptomatic Cartilage Defects of the Knee in a Randomized Controlled Trial Versus Microfracture

Daniel B. F. Saris, Johan Vanlauwe, Jan Victor, Miroslav Haspl, Michael Bohnsack, Yves Fortems, Bruno Vandekerckhove, K. Frederik Almqvist, Toon Claes, Frank Handelberg, Koen Lagae, Jan van der Bauwhede, Hilde Vandenneucker, K. Gie Auw Yang, Mislav Jelic, Rene Verdonk, Nancy Veulemans, Johan Bellemans and Frank P.

Luyten

Am J Sports Med 2008 36: 235

DOI: $10.1177 / 0363546507311095$

The online version of this article can be found at:

http://ajs.sagepub.com/content/36/2/235

\author{
Published by: \\ (3)SAGE \\ http://www.sagepublications.com \\ On behalf of: \\ 25 \\ American Orthopaedic Society for Sports Medicine
}

Additional services and information for The American Journal of Sports Medicine can be found at:

Email Alerts: http://ajs.sagepub.com/cgi/alerts

Subscriptions: http://ajs.sagepub.com/subscriptions

Reprints: http://www.sagepub.com/journalsReprints.nav

Permissions: http://www.sagepub.com/journalsPermissions.nav 


\section{Characterized Chondrocyte Implantation Results in Better Structural Repair When Treating Symptomatic Cartilage Defects of the Knee in a Randomized Controlled Trial Versus Microfracture}

Daniel B. F. Saris, ${ }^{\star a}$ MD, PhD, Johan Vanlauwe, ${ }^{b}$ MD, Jan Victor, ${ }^{c}$ MD, Miroslav Haspl, ${ }^{d}$ MD, PhD, Michael Bohnsack, ${ }^{e}$ MD, Yves Fortems, ${ }^{f}$ MD, Bruno Vandekerckhove, ${ }^{g}$ MD, K. Frederik Almqvist, ${ }^{h}$ MD, PhD, Toon Claes, ${ }^{i}$ MD, Frank Handelberg, ${ }^{j}$ MD, Koen Lagae, ${ }^{k}$ MD, Jan van der Bauwhede, 'MD, Hilde Vandenneucker, ${ }^{b}$ MD, K. Gie Auw Yang, ${ }^{a}$ MD, PhD, Mislav Jelic, ${ }^{\mathrm{d}}$ MD, PhD, Rene Verdonk, ${ }^{\mathrm{h}} \mathrm{MD}, \mathrm{PhD}$, Nancy Veulemans, ${ }^{\mathrm{m}} \mathrm{Ir}$, Johan Bellemans, ${ }^{b}$ MD, PhD, and Frank P. Luyten, ${ }^{n}$ MD, PhD

From the ${ }^{a}$ Department of Orthopedics, University Medical Center Utrecht, Utrecht, the Netherlands, ${ }^{b}$ Department Orthopedics, University Hospitals Leuven, Leuven, Belgium, ${ }^{c}$ Department of Orthopedics, AZ St Lucas Brugge, Brugge, Belgium, ${ }^{d}$ Department of Orthopaedic Surgery, School of Medicine, University of Zagreb, Zagreb, Croatia, ${ }^{e}$ Department of Orthopedic Surgery, Hannover Medical School, Hannover, Germany, ${ }^{f}$ Department of Orthopedics, AZ St Jozef, Malle, Belgium, ${ }^{g}$ Department of Orthopedics, AZ St Jan Brugge, Brugge, Belgium, 'h Department of Orthopedics, Ghent University Hospital, Ghent, Belgium, 'Department of Orthopedics, AZ St Elisabeth, Herentals, Belgium, 'Department of Orthopedics, Universitair Ziekenhuis Brussel, Brussels, Belgium, ' SPM Monica, Antwerp, Belgium, 'Department of Orthopedics, AZ Groeninge, Kortrijk, Belgium, ${ }^{m}$ Clinical Affairs, TiGenix NV, Leuven, Belgium, and ${ }^{n}$ Division of Rheumatology, Department of Musculoskeletal Sciences, University Hospitals, Katholieke Universiteit Leuven, Leuven, Belgium

Background: As the natural healing capacity of damaged articular cartilage is poor, joint surface injuries are a prime target for regenerative medicine. Characterized chondrocyte implantation uses an autologous cartilage cell therapy product that has been optimized for its biological potency to form stable cartilage tissue in vivo.

Purpose: To determine whether, in symptomatic cartilage defects of the femoral condyle, structural regeneration with characterized chondrocyte implantation is superior to repair with microfracture.

Study Design: Randomized controlled trial; Level of evidence, 1.

*Address correspondence to Daniel B. F. Saris, MD, PhD, PO Box 85500, 3508 GA Utrecht, the Netherlands (e-mail: d.saris@umcutrecht.nl).

Dr Vanlauwe and Dr Saris have contributed equally to this article and share first authorship.

One or more of the authors have declared a potential conflict of interest as specified in the AJSM Conflict of Interest statement: AO International reimbursement for educational activities, Smith \& Nephew orthopaedics reimbursement for educational activities, TiGenix reimbursement for educational activities, and Genzyme reimbursement for study design advice (D.B.F.S.); TiGenix stocks and warrants reimbursement for attending symposia (J.V.); TiGenix stocks (J.B.); Depuy International reimbursement for educational activities (Y.F.); reimbursement for study participation and case report form management (J.V.); TiGenix warrants, reimbursement for educational activities, Depuy J \& J reimbursement for educational activities (R.V.); TiGenix stocks reimbursement for attending research symposia, eg, for presenting at symposia (N.V.); cofounder of TiGenix and has a research contract with and stocks of TiGenix (F.P.L.).

The American Journal of Sports Medicine, Vol. 36, No. 2 DOI: 10.1177/0363546507311095

(C) 2008 American Orthopaedic Society for Sports Medicine 
Methods: Characterized chondrocyte implantation was compared with microfracture in patients with single grade III to IV symptomatic cartilage defects of the femoral condyles in a multicenter trial. Patients aged 18 to 50 years were randomized to characterized chondrocyte implantation $(n=57)$ or microfracture $(n=61)$. Structural repair was blindly assessed in biopsy specimens taken at 1 year using (1) computerized histomorphometry and (2) evaluation of overall histological components of structural repair. Clinical outcome was measured using the self administered Knee injury and Osteoarthritis Outcome Score. Adverse events were recorded throughout the study.

Results: Characterized chondrocyte implantation resulted in better structural repair, as assessed by histomorphometry $(P=.003)$ and overall histologic evaluation $(P=.012)$. Aspects of structural repair relating to chondrocyte phenotype and tissue structure were superior with characterized chondrocyte implantation. Clinical outcome as measured by the Knee injury and Osteoarthritis Outcome Score at 12 to 18 months after characterized chondrocyte implantation was comparable with microfracture at this stage. Both treatment groups had a similar mean baseline overall Knee injury and Osteoarthritis Outcome Score (56.30 \pm 13.61 and $59.53 \pm 14.95$ for microfracture and characterized chondrocyte implantation, respectively), which increased in both groups to $70.56 \pm 12.39$ and $72.63 \pm 15.55$ at 6 months, $73.26 \pm 14.66$ and $73.10 \pm 16.01$ at 12 months, and $74.73 \pm 17.01$ and $75.04 \pm$ 14.50 at 18 months, respectively. Both techniques were generally well tolerated; the incidence of adverse events after characterized chondrocyte implantation was not markedly increased compared with that for microfracture.

Conclusion: One year after treatment, characterized chondrocyte implantation was associated with a tissue regenerate that was superior to that after microfracture. Short-term clinical outcome was similar for both treatments. The superior structural outcome may result in improved long-term clinical benefit with characterized chondrocyte implantation. Long-term follow-up is needed to confirm these findings.

Keywords: chondrocyte; chondral; regenerative medicine; autologous chondrocyte implantation (ACl); characterized chondrocyte implantation (CCl); cell therapy product; microfracture; cartilage repair

Regenerative medicine holds great promise for the reconstitution of damaged tissues and organs. Articular cartilage injuries are a prime target for regenerative techniques, as spontaneous healing is poor. When untreated, cartilage injuries predispose to osteoarthritis, which is a major cause of disability and represents a huge socioeconomic burden to society. ${ }^{8,13,17,40}$

The aim of treatment for cartilage damage is to restore normal, long-term, and pain-free motion of the joint by promoting the formation of repair tissue with the structure and durability of natural hyaline-like articular cartilage., ${ }^{7,21,33}$ Marrow stimulation techniques (such as microfracture $[\mathrm{MF}]$ ), mosaicplasty, and autologous chondrocyte implantation (ACI) are currently used joint-resurfacing techniques. Although studies have been conducted to evaluate their efficacy, the methodological quality has been generally $\operatorname{low}^{22}$ and the results conflicting. Consequently, no evidence-based consensus has emerged regarding the best treatment option.

Autologous chondrocyte implantation was one of the first techniques to introduce the principle of regenerative medicine. It is a 2-step procedure with a cartilage harvest during arthroscopy and implantation of ex vivo-expanded chondrocytes during arthrotomy 4 to 5 weeks later. ${ }^{23}$ Since the first publication of this technique 13 years ago, ${ }^{3}$ more than 12000 patients have undergone ACI. ${ }^{15}$ Convincing clinical evidence regarding its efficacy and safety is still lacking, ${ }^{22}$ with only one level 1 study published to date. ${ }^{24}$ Although hyaline-like repair tissue and good long-term results have been demonstrated after conventional ACI, ${ }^{31}$ some studies have shown ACI to be associated with the formation of fibrocartilage or mixed hyaline/fibrocartilage. ${ }^{2,19}$ Expansion of autologous cells in conventional ACI leads to dedifferentiation and loss of chondrogenic capacity. ${ }^{1,9,11}$ Characterized chondrocyte implantation (CCI) has been developed to improve the results of articular regeneration with chondrocyte cell therapy through the use of a cell population capable of making stable hyaline-like cartilage in vivo. ${ }^{9-11}$ Characterized chondrocytes are an expanded population of chondrocytes that expresses a marker profile (a gene score) predictive of the capacity to form hyaline-like cartilage in vivo in a consistent and reproducible manner. This marker profile has been used to optimize the cell expansion procedures for preserving phenotypic characteristics and biological activity and therefore optimizes the potency of each cell batch. The expansion procedure and the process of gene scoring are designed to ensure a higher degree of homogeneity in the cartilage-forming capacity of the individual expanded cell populations. ${ }^{10}$

This prospective randomized controlled trial (RCT) was designed to compare the efficacy and safety of CCI versus MF in the repair of single symptomatic cartilage defects of the femoral condyle. Microfracture was chosen as the comparator as it is the most frequently used first-line treatment for this condition. ${ }^{14}$ The dual primary aims of the study were (1) to demonstrate superiority of CCI over MF in the overall quality of structural regeneration of the articular tissue at 12 months posttreatment (by histomorphometry and an overall histology assessment score) and (2) to demonstrate a clinical outcome as assessed by the overall Knee injury and Osteoarthritis Outcome Score (KOOS) at 12 to 18 months after treatment that was at least comparable in both treatment groups.

\section{MATERIALS AND METHODS}

\section{Study Population}

This multicenter study involved 13 surgeons at 13 orthopaedic centers in Belgium, the Netherlands, Germany, and Croatia. The study conformed to the Declaration of Helsinki and was conducted according to the International Conference on Harmonization Guidelines for Good Clinical Practice. The protocol and all 
TABLE 1

Inclusion and Exclusion Criteria ${ }^{a}$

Inclusion Criteria
Patients provided written informed consent
Patients were aged between 18 and 50 years
Symptomatic cartilage single lesions of the femoral condyles
Lesion on the femoral condyle between 1 and $5 \mathrm{~cm}^{2}$
Patients agreed to actively participate in a strict rehabilitation
protocol and follow-up program
Patients agreed to use only paracetamol mono or combination
preparation (maximum dose of 4 g/d) and nonsteroidal
anti-inflammatory drugs during the study and to
discontinue this medication 2 weeks before the baseline
visit and the follow-up visits at 8 weeks and $3,6,9$, and
12 months after surgery; however, the use of paracetamol
mono preparation (maximum dose of 4 g/d) was allowed up
to 1 week before the baseline visit and the follow-up visits
at 8 weeks and $3,6,9$, and 12 months after surgery
Male or female patients; women of childbearing age
had to use a proven method to prevent pregnancy

Exclusion Criteria

Participation in concurrent trials

Participation in previous trial within 3 months

Patients with human immunodeficiency virus, hepatitis, or syphilis (protocol amendment 4 removed the requirement that patients with cytomegalovirus were excluded)

Malignancy

Alcohol and drug (medication) abuse

Poor general health condition as judged by investigator

Presence of a clinically relevant cartilage lesion on the patella (second lesion)

Patellofemoral cartilage lesion

Osteochondritis dissecans: recent (within 1 year before baseline); depth of lesion $>0.5 \mathrm{~cm}$; subchondral sclerosis

Advanced osteoarthritis (as defined by Radiographic Atlas of Osteoarthritis, grade 2-3)

Known allergy to penicillins and gentamicin (or presence of multiple severe allergies)

Complex ligamentous instability of the knee

Meniscal transplant (previous/present)

Meniscal suture with meniscal arrows ipsilaterally (present or previous if not resorbed)

Meniscal resection if:

Less than 1 year before baseline: lateral meniscal resection or medial meniscal resection of more than $50 \%$

More than 1 year before baseline: ipsilateral meniscal resection of more than $50 \%$, contralateral meniscal resection of more than $50 \%$ if ipsilateral meniscus is not intact, or combination of medial and lateral meniscus resection and 1 or both $>50 \%$ resected

Varus or valgus malalignment exceeding $5^{\circ}$ (kissing lesions out)

Mosaicplasty (osteochondral autograft transfer system)

Microfracture performed less than 1 year before baseline

Received hyaluronic acid intra-articular injections into the afflicted knee within the last 6 months of baseline

Taking specific osteoarthritis drugs, such as chondroitin sulfate, diacerein, n-glucosamine, piascledine, and capsaicin, within 2 weeks of the baseline visit

Corticosteroid therapy by systemic or intra-articular route within the last month of baseline or intramuscular or oral corticosteroids within the last 2 weeks of baseline

Chronic use of anticoagulants

Uncontrolled diabetes

Any concomitant painful or disabling disease of the spine, hips, or lower limbs that would interfere with evaluation of the afflicted knee

Any clinically significant or symptomatic vascular or neurologic disorder of the lower extremities

Any evidence of the following diseases in the target joint: septic arthritis; inflammatory joint disease; gout; recurrent episodes of pseudogout; Paget disease of bone; ochronosis; acromegaly; hemochromatosis; Wilson disease; primary osteochondromatosis; heritable disorders; collagen gene mutations

Current diagnosis of osteomyelitis

A blood result showing liver enzymes (serum glutamic oxaloacetic transaminase, serum glutamic pyruvic transaminase, alkaline phosphatase) of more than 2 times the upper limit of normal or any other result that in the clinical investigator's mind is clinically important C-reactive protein level greater than $10 \mathrm{mg} / \mathrm{L}$

\footnotetext{
${ }^{a}$ Patients with ACL injuries were also included in the study as long as the injury was repaired (patients in both treatment groups). Prior debridement or lavage was also allowed.

amendments were approved by the ethics review board at each participating center, and all patients gave written informed consent to participate.

The first patient was enrolled in the study on February 21, 2002, and the last patient completed the 18-month

assessments on July 25, 2006. Eligible patients were aged between 18 and 50 years, had a symptomatic single cartilage lesion of the femoral condyles (between 1 and $5 \mathrm{~cm}^{2}$ ) (Tables 1 and 2), and agreed to actively participate in a strict rehabilitation and follow-up program.
} 
TABLE 2

Baseline Characteristics of the Patients ${ }^{a}$

\begin{tabular}{|c|c|c|}
\hline General Demographics & Microfracture $(\mathrm{n}=61)$ & CCI $(n=57)$ \\
\hline Age, y & $33.9 \pm 8.6$ & $33.9 \pm 8.5$ \\
\hline \multicolumn{3}{|l|}{ No. in each age category $(\%)$} \\
\hline$\geq 40$ years & $18(30)$ & $17(30)$ \\
\hline Height, cm & $177.0 \pm 8.5$ & $176.5 \pm 10.8$ \\
\hline Weight, kg & $80.6 \pm 13.3$ & $78.3 \pm 13.9$ \\
\hline Female & $20(33)$ & $22(39)$ \\
\hline \multicolumn{3}{|l|}{ No. in each body mass index category (\%) } \\
\hline$\leq 25 \mathrm{~kg} / \mathrm{m}^{2}$ & $31(51)$ & $28(49)$ \\
\hline$>25$ to $\leq 30 \mathrm{~kg} / \mathrm{m}^{2}$ & $24(39)$ & $26(46)$ \\
\hline$>30 \mathrm{~kg} / \mathrm{m}^{2}$ & $6(9.8)$ & $3(5.3)$ \\
\hline \multicolumn{3}{|l|}{ Knee condition at screening } \\
\hline No. with normal opposite knee (\%) & $50(82)$ & $38(67)$ \\
\hline No. with any previous knee surgery ${ }^{b}(\%)$ & $47(77)$ & $50(88)$ \\
\hline \multicolumn{3}{|l|}{ No. of previous knee surgeries (\%) } \\
\hline 0 & $14(23)$ & $7(12)$ \\
\hline 1 & $34(56)$ & $29(51)$ \\
\hline$\geq 2$ & $13(21)$ & $21(37)$ \\
\hline \multicolumn{3}{|l|}{ Femoral condyle at baseline arthroscopy } \\
\hline No. with single cartilage lesion $(\%)$ & $59(97)$ & $56(98)$ \\
\hline Defect size postdebridement, $\mathrm{cm}^{2}$ & $2.4 \pm 1.2$ & $2.6 \pm 1.0$ \\
\hline No. with cartilage lesion grade IV (\%) & $45(74)$ & $47(82)$ \\
\hline No. with associated lesions (\%) & $15(25)$ & $17(30)$ \\
\hline \multicolumn{3}{|c|}{ No. of concomitant lesions treated during the study (\%) } \\
\hline
\end{tabular}

${ }^{a}$ Values are means \pm SDs (unless stated otherwise). CCI, characterized chondrocyte implantation; ML, meniscal lesion.

${ }^{b}$ In the CCI group, 5 patients had previous microfracture and 3 had previous subchondral drilling. In the microfracture group, 1 patient had previous microfracture and 2 had previous subchondral drilling. In addition, 1 patient in each group had previous abrasion arthroplasty.

${ }^{c}$ In the microfracture group, 5 of the 6 patients categorized as being treated for a meniscal lesion during the study received only shaving or debridement rather than a surgical resection.

\section{Study Design and Treatment Randomization}

After screening, all patients underwent arthroscopy, during which the knee and the suspected cartilage defect were inspected. At this time, patients were randomly allocated to either CCI or MF using a minimization method ${ }^{32}$ to achieve treatment balance with respect to operating surgeon, location of lesion, and presence or absence of associated lesions.

For both procedures, surgeons and study team personnel were trained extensively in the standardization of surgical technique and study procedures, including the use of CCI (ChondroCelect, TiGenix NV, Haasrode, Belgium). Patients allocated to the MF group were treated according to the technique described by Steadman et al. ${ }^{39}$ Those allocated to the CCI group were treated as described by Brittberg et al. ${ }^{3}$ For CCI, cartilage biopsies were taken during arthroscopy from the medial femoral condyle rim outside of the loaded area with a specially designed 4-mm-wide, sharp Wiberg raspatorium. The biopsy sample included only cartilage; subchondral bone was not penetrated. Up to 3 cartilage slices, 7 to $8 \mathrm{~mm}$ long and a few millimeters wide, were obtained. Chondrocytes were isolated from the cartilage biopsy specimen and expanded ex vivo in a Good Manufacturing Practice-approved facility, and their phenotype was characterized. Each batch of ChondroCelect was quantitatively graded using the ChondroCelect score (CC score) based on the quantitative gene expression of a selection of positive and negative markers developed to predict the cells' ability to form stable hyaline cartilage in vivo. ${ }^{9-11}$ Characterized chondrocyte implantation was performed 27 days ( \pm 5 days) after chondrocyte harvest. ChondroCelect was administered at a target dose of between 0.8 and $1 \times 10^{6}$ cells $/ \mathrm{cm}^{2}$, with the characterized cells implanted beneath an autologous periosteal flap procured from the proximal medial tibia.

After surgery, both groups of patients were enrolled in an identical standardized rehabilitation program and attended regular follow-up visits at the clinic. Patients 
were to refrain from taking pain relief medications for 2 weeks before baseline and 2 weeks before each subsequent study assessment. Investigators were responsible for the presurgical assessments, surgery, and end point biopsy. A coordinating surgeon ensured surgical standardization during cartilage harvest, treatment, and end point biopsy, and an independent evaluator at each study center was responsible for all postsurgical evaluations.

\section{Rehabilitation Program}

All patients were required to participate in a rehabilitation program that commenced on the day of MF or CCI and continued either at the hospital or at home, according to a strict schedule. The rehabilitation program was based on the different phases of wound healing, being especially focused on graft protection and wound control. Restrictions on weightbearing and range of motion were imposed. Patients were nonweightbearing for the first 2 weeks after surgery; thereafter, weightbearing increased to 10 to $15 \mathrm{~kg}$ for the third week after surgery and to 15 to $30 \mathrm{~kg}$ for the fourth to sixth weeks after surgery. After 6 weeks, progressive load bearing, as tolerated by the patient, was permitted for patients in both treatment groups. Patients were required to wear an unloader brace for the first 8 weeks after surgery.

A coordinating physical therapist was responsible for training of all hospital and personal physical therapists whom the patient visited. All physical therapists responsible for implementing the rehabilitation program at the investigational sites received initial training, coordination, ongoing training, and feedback from the coordinating physical therapist during the study. The coordinating physical therapist was also responsible for follow-up and feedback to program physical therapists based on weekly logs for all patients.

After the arthroscopy for end point biopsy, no full impact was allowed for the first 6 weeks. Patients were permitted only partial weightbearing (with 2 crutches) for the first 3 days; thereafter, if the patients were pain free, had no swelling, and had good local muscle function, they could be weaned off crutches so that during weeks 2 to 4 after arthroscopy, the patients could gradually increase their activities of daily living (although no full-impact exercises were permitted). During weeks 4 to 8 after arthroscopy, patients were to gradually increase impact activities to pre-arthroscopy levels.

\section{Histologic Analysis}

Cylindrical full-thickness cartilage biopsy specimens of 2-mm diameter were obtained arthroscopically from the center of the repair tissue with a custom-made biopsy needle, 12 months after treatment. The specimens were fixed in paraformaldehyde before being embedded in paraffin and sectioned at a thickness of 2 to $5 \mu \mathrm{m}$. Sections were stained with hematoxylin and eosin, safranin $\mathrm{O}$, and anticollagen II antibodies.

Histomorphometry was performed at the Institute for Pathology, University of Bern, Switzerland, by independent personnel blinded to treatment allocation. Stained sections were digitized using a digital camera (Zeiss,
Oberkochen, Germany), and the stain signal for safranin $O$ and anticollagen II antibody was determined using Image Pro Plus software (Media Cybernetics, Silver Spring, Md). The surface area of the cartilage was estimated using a calibrated macro. The extent of staining with safranin $\mathrm{O}$ and anticollagen II antibody was expressed as a ratio of the total surface area of the cartilage, ${ }^{30}$ and the ratios were summed.

The overall histology assessment scores were determined by 2 blinded independent histopathologists specializing in cartilage histology, who assessed the quality of cartilage repair. The histopathologists also evaluated individual components of cartilage repair related to chondrocyte phenotype (matrix-staining metachromasia, cell morphologic characteristics), tissue structure (tissue morphologic characteristics, calcification front/tidemark, basal integration, mid/deep zone assessment, surface architecture, surface/superficial assessment), and other possible negative features of cartilage repair (vascularization, inflammation, subchondral bone abnormalities/cellular infiltration, abnormal calcification/debris). Each item was scored using a 100-mm visual analog scale (VAS).

The histopathologists responsible for the histology scoring were trained to grade sections in a consistent manner before performing the analysis of the sections for this clinical trial.

\section{Assessment of Clinical Outcome}

Clinical outcome was assessed using the self administered KOOS questionnaire, consisting of 5 subdomains: symptoms/ stiffness, pain, activities of daily living, function in sports and recreational activities, and quality of life. Scores for the summed (overall KOOS) and individual subdomains were transformed to a scale of 0 to 100 , with 0 representing extreme knee problems and 100 representing no knee problems. ${ }^{36}$ The sports domain was not included in the overall KOOS because too many items were not reported by the patients. According to the prespecified statistical analysis plan, if more than 2 items had not been completed, the response was considered invalid and no score calculated for that specific domain of the KOOS (see Table 3).

\section{Safety}

Safety was assessed by physical examinations, vital signs, electrocardiograms, clinical laboratory tests, and continuous monitoring of adverse events (AEs).

All AEs experienced by the patient from the time of screening visit 1 (day 28) until completion of both the initial 12-month and extension studies were recorded in the case report form $(\mathrm{CRF})$.

Details of the AEs that were to be recorded in the CRF included a brief description, severity, causality, action taken, start and stop dates, outcome, and seriousness.

Investigators were required to assign a causal relationship to the study procedure (CCI or MF). It should be noted that relatedness in the context of the initial 12-month study refers to whether an $\mathrm{AE}$ was considered to be related to the combination of both surgical procedure and investigational medicinal product (ChondroCelect) administration for 
TABLE 3

Overall KOOS and Individual KOOS Subdomains at Baseline and at 6, 12, and 18 Months After Surgery ${ }^{a}$

\begin{tabular}{|c|c|c|c|c|c|c|c|c|}
\hline \multirow{2}{*}{$\begin{array}{l}\text { KOOS and } \\
\text { Subdomains }\end{array}$} & \multicolumn{4}{|c|}{ CCI } & \multicolumn{4}{|c|}{ Microfracture } \\
\hline & Baseline & Month 6 & Month 12 & Month 18 & Baseline & Month 6 & Month 12 & Month 18 \\
\hline Overall KOOS ${ }^{b}$ & $\begin{array}{c}56.30 \pm 13.61 \\
(\mathrm{n}=51)\end{array}$ & $\begin{array}{c}70.56 \pm 12.39 \\
(\mathrm{n}=51)\end{array}$ & $\begin{array}{c}73.26 \pm 14.66 \\
(\mathrm{n}=51)\end{array}$ & $\begin{array}{c}74.73 \pm 17.01 \\
(\mathrm{n}=44)\end{array}$ & $\begin{array}{c}59.53 \pm 14.95 \\
(\mathrm{n}=61)\end{array}$ & $\begin{array}{c}72.63 \pm 15.55 \\
(\mathrm{n}=59)\end{array}$ & $\begin{array}{c}73.10 \pm 16.01 \\
(\mathrm{n}=57)\end{array}$ & $\begin{array}{c}75.04 \pm 14.50 \\
(\mathrm{n}=51)\end{array}$ \\
\hline $\mathrm{ADL}$ & $\begin{array}{c}68.94 \pm 18.55 \\
(\mathrm{n}=51)\end{array}$ & $\begin{array}{c}83.69 \pm 13.83 \\
\quad(\mathrm{n}=51)\end{array}$ & $\begin{array}{c}85.32 \pm 17.07 \\
\quad(\mathrm{n}=51)\end{array}$ & $\begin{array}{c}85.03 \pm 18.18 \\
(\mathrm{n}=44)\end{array}$ & $\begin{array}{c}71.85 \pm 18.65 \\
(\mathrm{n}=61)\end{array}$ & $\begin{array}{c}84.58 \pm 15.21 \\
\quad(\mathrm{n}=59)\end{array}$ & $\begin{array}{c}83.58 \pm 16.92 \\
\quad(\mathrm{n}=56)\end{array}$ & $\begin{array}{c}87.26 \pm 14.20 \\
(\mathrm{n}=50)\end{array}$ \\
\hline Pain & $\begin{array}{c}62.05 \pm 18.73 \\
(\mathrm{n}=51)\end{array}$ & $\begin{array}{c}77.12 \pm 13.79 \\
\quad(\mathrm{n}=51)\end{array}$ & $\begin{array}{c}78.85 \pm 18.80 \\
(\mathrm{n}=51)\end{array}$ & $\begin{array}{c}79.55 \pm 18.75 \\
(\mathrm{n}=44)\end{array}$ & $\begin{array}{c}65.47 \pm 17.13 \\
\quad(\mathrm{n}=61)\end{array}$ & $\begin{array}{c}79.52 \pm 15.65 \\
(\mathrm{n}=59)\end{array}$ & $\begin{array}{c}78.79 \pm 16.79 \\
(\mathrm{n}=57)\end{array}$ & $\begin{array}{c}81.20 \pm 15.07 \\
\quad(\mathrm{n}=51)\end{array}$ \\
\hline $\begin{array}{c}\text { Symptoms/ } \\
\text { stiffness }\end{array}$ & $\begin{array}{c}65.03 \pm 16.37 \\
(\mathrm{n}=51)\end{array}$ & $\begin{array}{c}75.35 \pm 14.36 \\
(\mathrm{n}=51)\end{array}$ & $\begin{array}{c}79.23 \pm 12.15 \\
(\mathrm{n}=51)\end{array}$ & $\begin{array}{c}79.38 \pm 16.26 \\
(\mathrm{n}=44)\end{array}$ & $\begin{array}{c}68.12 \pm 16.76 \\
\quad(\mathrm{n}=61)\end{array}$ & $\begin{array}{c}79.08 \pm 15.60 \\
(\mathrm{n}=59)\end{array}$ & $\begin{array}{c}79.14 \pm 15.37 \\
(\mathrm{n}=57)\end{array}$ & $\begin{array}{c}79.81 \pm 14.43 \\
(\mathrm{n}=51)\end{array}$ \\
\hline QoL & $\begin{array}{c}29.17 \pm 14.18 \\
(\mathrm{n}=51)\end{array}$ & $\begin{array}{l}46.08 \pm 18.54 \\
\quad(\mathrm{n}=51)\end{array}$ & $\begin{array}{l}49.63 \pm 19.50 \\
\quad(\mathrm{n}=51)\end{array}$ & $\begin{array}{c}54.97 \pm 22.34 \\
(\mathrm{n}=44)\end{array}$ & $\begin{array}{c}32.68 \pm 18.69 \\
\quad(\mathrm{n}=61)\end{array}$ & $\begin{array}{c}47.35 \pm 22.96 \\
\quad(\mathrm{n}=59)\end{array}$ & $\begin{array}{c}49.89 \pm 23.46 \\
(\mathrm{n}=55)\end{array}$ & $\begin{array}{c}52.45 \pm 23.05 \\
(\mathrm{n}=51)\end{array}$ \\
\hline
\end{tabular}

${ }^{a}$ Values are means \pm SDs. ADL, activities of daily living; CCI, characterized chondrocyte implantation; KOOS, Knee injury and Osteoarthritis Outcome Score; QoL, quality of life.

${ }^{b}$ Sports domain not included because too many items were not reported by the patients.

patients in the CCI group and surgical procedure alone for patients who were assigned to the MF group. All AEs were monitored until there was a return to normal or until the condition became stable or had resolved. The patient continued to be followed after completing the study if any $\mathrm{AE}$ had not stabilized or resolved at the end of the study period.

\section{Statistical Analysis}

Analyses were performed according to the intention-totreat principle. Safety analyses included all randomized patients who received any study treatment (harvest biopsy or MF). Efficacy analyses were performed on all randomized patients who did not withdraw before completion of the surgical treatment.

The sample size for this study was determined using a categorization (success/failure) whereby the presence of hyaline or hyaline-like tissue was recorded as a success and fibrocartilage or noncartilage as a failure. It was assumed that $30 \%$ of patients allocated to MF would be categorized as a success and that an improvement in this success rate to $60 \%$ success with CCI would constitute a clinically significant improvement. On this basis, with $90 \%$ power and using a $2.5 \%$ onesided test, it was calculated that a total enrollment of 112 patients (56/group) would be required.

The sum of histomorphometric scores on safranin $\mathrm{O}$ and collagen II staining and the mean overall histology assessment score at 12 months were analyzed using fixed-effects analysis of covariance, with treatment, location of lesions, and associated lesion as categorical variables and age as a covariate. The differences in adjusted means for the histomorphometric sum of the safranin $\mathrm{O}$ and collagen II end point, and for the overall histology assessment score, were tested independently for equality to zero. Multiple comparison adjustments were controlled at a significance level of .05 using the Hochberg procedure. ${ }^{18}$

The change from baseline in KOOS averaged over 12 and 18 months was analyzed using the same method as for structural repair, with baseline KOOS added to the analysis of covariance model as a continuous variable. Noninferiority for the overall KOOS was defined as having been demonstrated if the lower end of the $95 \%$ confidence interval for the difference between adjusted means in the 2 treatment groups was above -9 percentage points. ${ }^{12,20,24}$ For missing KOOS domain scores due to reintervention of the index lesion (ie, treatment failure), a last observation carried forward approach was used, whereby missing values were replaced with the last previous nonmissing value.

Adverse events that occurred with a frequency of $5 \%$ or more in either treatment group were compared using the Fisher exact test.

\section{RESULTS}

\section{Patient Enrollment and Baseline Characteristics}

Of the 118 patients enrolled in the study, 57 were randomized to CCI and 61 to MF. Patient disposition and the numbers of patients who contributed data to the structural repair and clinical outcome analyses are presented in Figure 1 . The study was unblinded after the last patient treated had been followed up for 18 months.

Table 2 illustrates that both treatment groups were generally well matched regarding patient baseline characteristics. Proportionally more patients in the CCI group versus the MF group had undergone previous knee surgery (50/57 [88\%] vs 47/61 [77\%] patients), in particular, marrow stimulation, and had undergone 2 or more previous knee surgeries (21/57 [37\%] vs 13/61 [21\%]). The median duration of symptoms of patients in the CCI group was slightly longer than that of the MF group (1.97 vs 1.57 years). Concomitant lesions treated during the study were mainly ACL lesions (3/57 [5.3\%] in the CCI group vs 4/61 [6.6\%] in the MF group), meniscal lesions (0/57 in the CCI group vs $6 / 61$ [9.8\%] in the MF group), or a combination of ACL and meniscal lesion (1/57 [1\%] in the CCI group vs $1 / 61$ [1.6\%] in the MF group). In the MF group, 5 of 6 


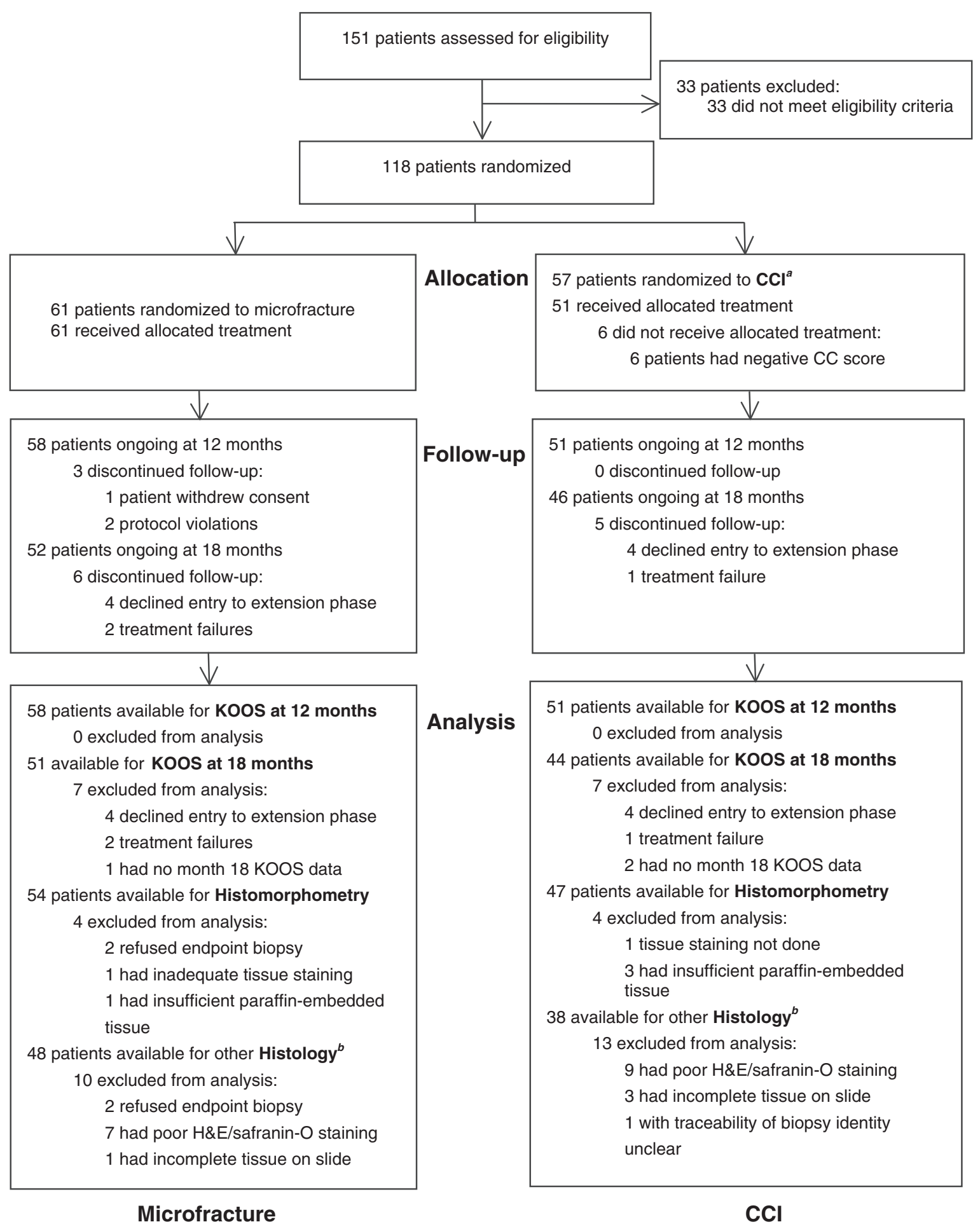

Figure 1. Enrollment and outcomes. CC, ChondroCelect; CCl, characterized chondrocyte implantation; KOOS, Knee injury and Osteoarthritis Outcome Score. ${ }^{a}$ ChondroCelect is an expanded population of chondrocytes that expresses a marker profile (a gene score) predictive of the capacity to form hyaline-like cartilage in vivo in a consistent and reproducible manner. This marker profile has been used to optimize the cell expansion procedures for preserving biological activity and therefore optimizes the potency of each cell batch. This process of gene scoring ensures a higher degree of homogeneity in the cartilage-forming capacity of the individual expanded cell populations. The ChondroCelect score (CC score), ranging from -6 to +6 ( 4 positive and 2 negative genetic markers), performed at the end of the culturing process is considered a potency assay. Using a cutoff (CC score $\geq$ 0 ) as a release criterion confirms that only chondrocytes having a specific genetic profile related to a high cartilage-forming capacity are implanted in the patient. ${ }^{b}$ Note that not all samples were evaluated for all 14 histology scales (eg, overall histology assessment score: $\mathrm{CCl}, \mathrm{n}=36$; microfracture, $\mathrm{n}=46$; inflammation scale: $\mathrm{CCl}, \mathrm{n}=28$; microfracture, $\mathrm{n}=36$ ). 
A

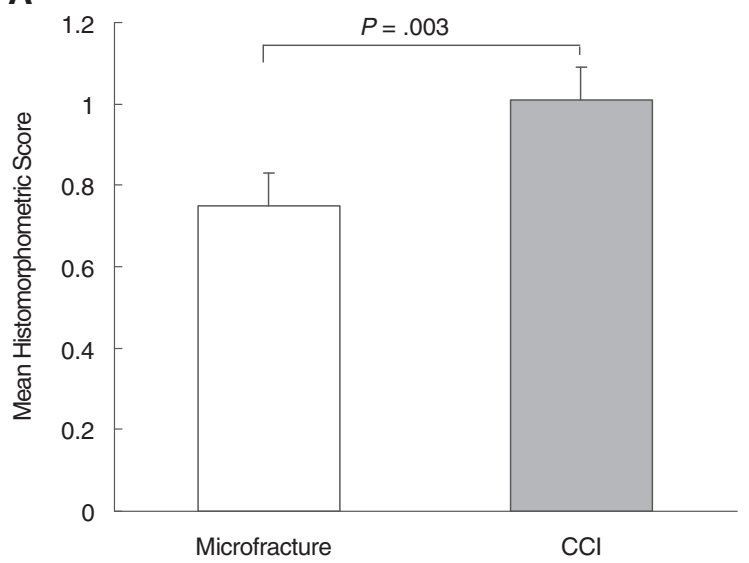

Computer-Assisted Histomorphometry

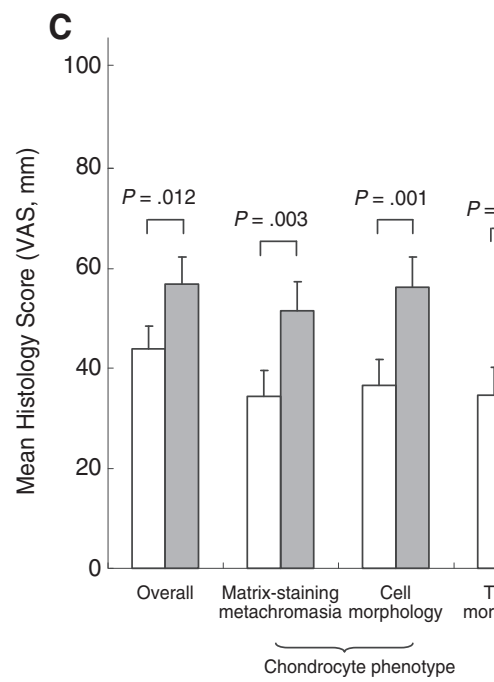

B

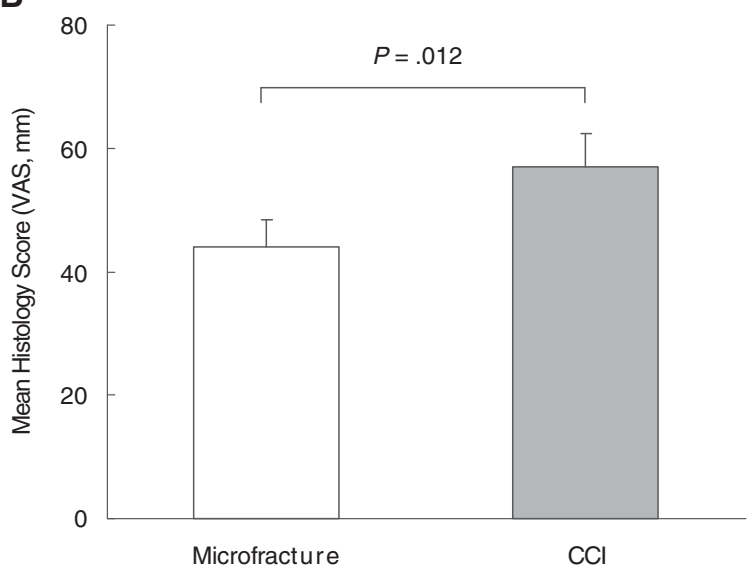

Overall Histology Assessment

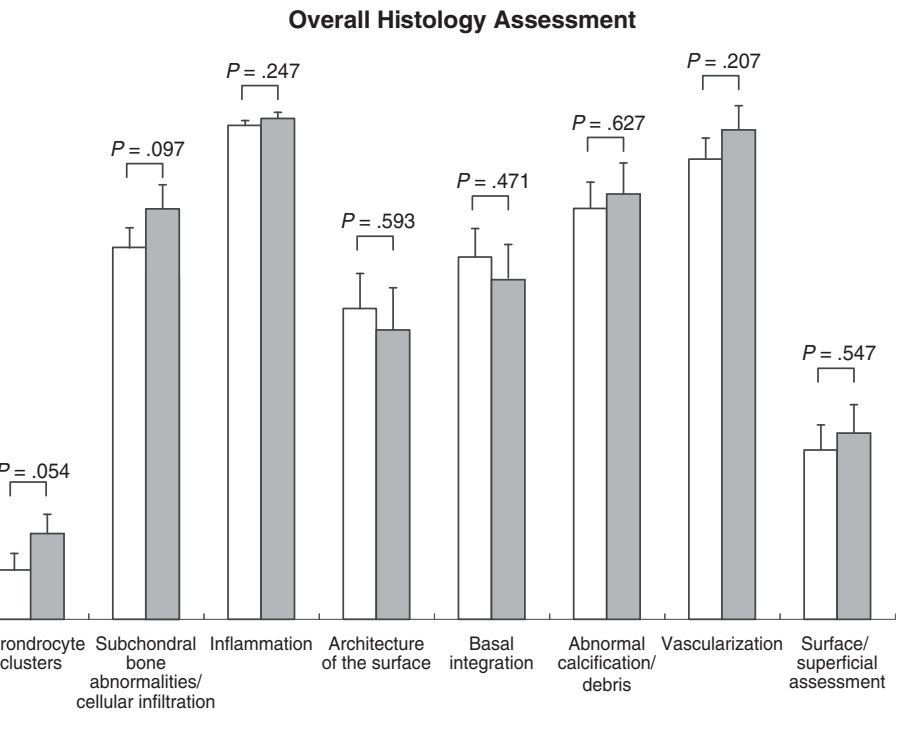

Histological Components of Structural Repair

Figure 2. Effect of $\mathrm{CCl}$ as compared with microfracture on structural repair determined from histologic evaluation of biopsy specimens taken 12 months after surgery. In all panels, the clear bars represent microfracture, and the shaded bars represent CCl. All values presented are adjusted means ( \pm standard error). $P$ values are provided for differences in adjusted means between $\mathrm{CCl}$ and microfracture. Panel A demonstrates the computer automated measurement of cartilage surface area. In panel B, columns represent the mean scores from 2 histopathologists, blinded to treatment allocation, for the overall histology assessment score (scored on 100-mm VAS, on which a score of $0 \mathrm{~mm}$ was bad and $100 \mathrm{~mm}$ was good). In panel C, the mean scores from 2 histopathologists, blinded to treatment allocation, for other structural components (scored on 100-mm VAS, on which a score of $0 \mathrm{~mm}$ indicates a poor repair result and $100 \mathrm{~mm}$ is an ideal repair result). CCl, characterized chondrocyte implantation; VAS, visual analog scale.

patients categorized as being treated for a meniscal lesion during the study received only shaving or debridement rather than surgical resection.

\section{Structural Outcome}

Structural regeneration of the articulating cartilage surface was better after CCI than after MF, as demonstrated by the adjusted mean sum of ratios for safranin $\mathrm{O}$ and collagen II staining, determined by histomorphometry, being statistically significantly higher $(P=.003)$ for the CCI group than for the MF group (Figure 2A). This mean histomorphometric score, a measure of proteoglycan and collagen II, is considered a quantitative and objective measure of good structural regeneration of cartilage tissue. ${ }^{30}$

The adjusted mean overall histology assessment score was statistically significantly higher $(P=.012)$ for the CCI treatment group than for the MF group (Figure 2B). Interreader agreement for the overall histology assessment scores showed a Pearson correlation coefficient of .78.

Adjusted mean scores for components of structural repair relating to chondrocyte phenotype and some components relating to tissue structure were also significantly higher for the CCI group than for the MF group (see Figure 2C). 

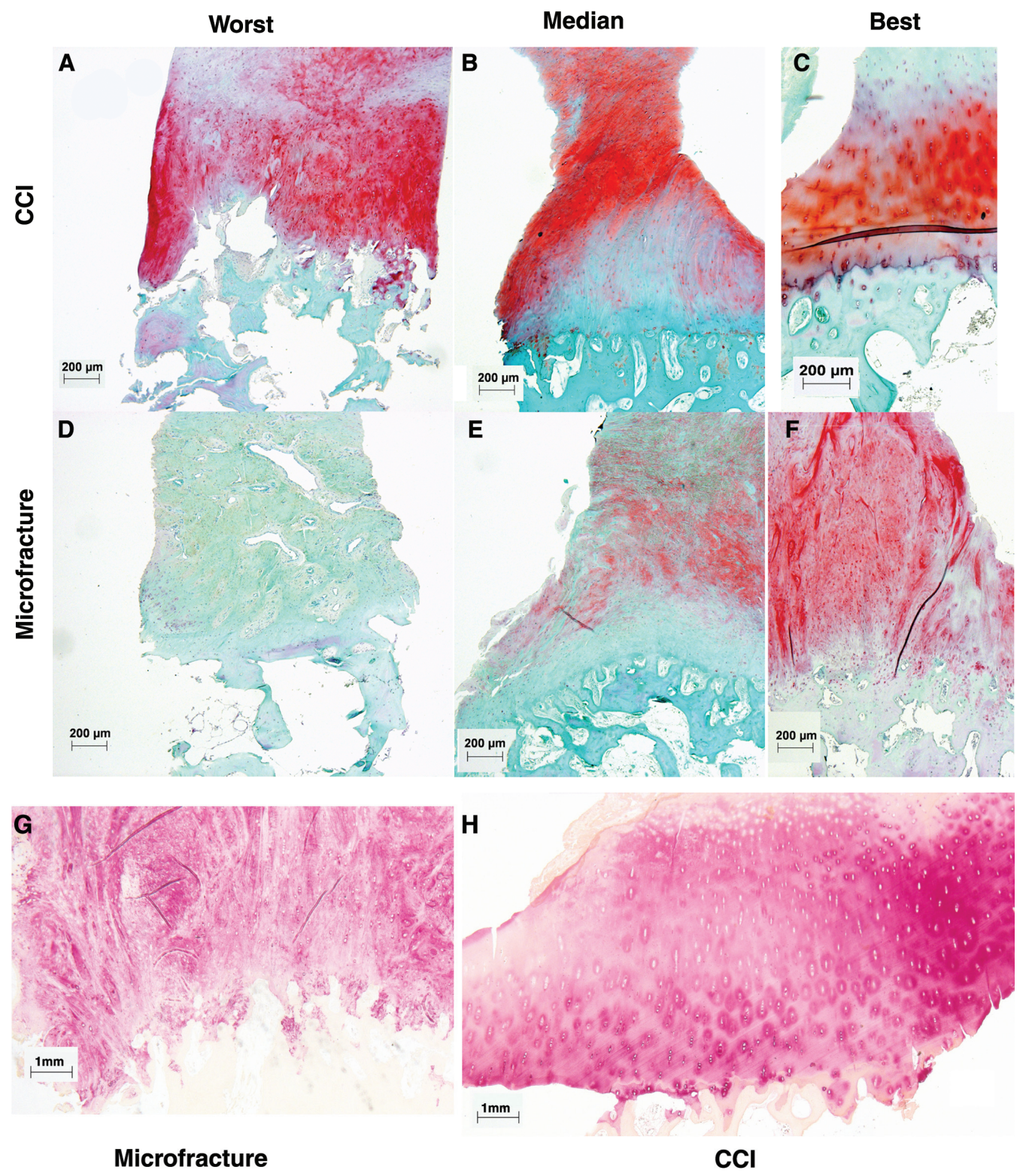

Figure 3. Effect of $\mathrm{CCl}$ compared with microfracture on safranin $\mathrm{O}(\mathrm{A}-\mathrm{F})$ and anti collagen II antibody (G-H) staining of 12-month biopsy specimens. Sections presented from the $\mathrm{CCl}$ and microfracture treatment groups have been selected based on the overall histology assessment scores and are representative of the worst $(A, D)$, median $(B, E)$, and best $(C, F)$ images. There is a clear morphological superiority of the tissue regeneration after $\mathrm{CCl}(\mathrm{H})$ as shown in the collagen type II immunostaining when compared with that of the scar tissue formation after microfracture (G). CCl, characterized chondrocyte implantation.

The statistically significant differences between the 2 treatment groups in the quality of tissue restoration are strikingly reflected in the repair scar tissue that may be observed after MF compared with the obvious cartilaginous restoration after CCI, as demonstrated in biopsy specimens stained with safranin $\mathrm{O}$ and collagen II (Figure 3).

\section{Clinical Outcome}

The adjusted means for the change from baseline to the mean of 12 and 18 months in overall KOOS and the subdomains of pain, symptoms/stiffness, activities of daily living, and quality of life were also similar for the 2 treatment groups (see Figure 4 and Table 3). The lower limit of the
95\% confidence interval for the difference between adjusted means (ie, -3.28 percentage points) in overall KOOS was above the predefined delta for noninferiority testing, confirming CCI being comparable to MF at this stage.

\section{Safety}

Similar proportions of patients recorded 1 or more treatmentemergent (postrandomization) AEs in the CCI group compared with the MF group (50/57 [88\%] vs 50/61 [82\%] patients). The majority of AEs were of mild or moderate intensity, and the proportion of patients who experienced 1 or more severe AEs was similar in both treatment groups (7/57 [12\%] vs 8/61 [13\%] patients in the CCI and MF 


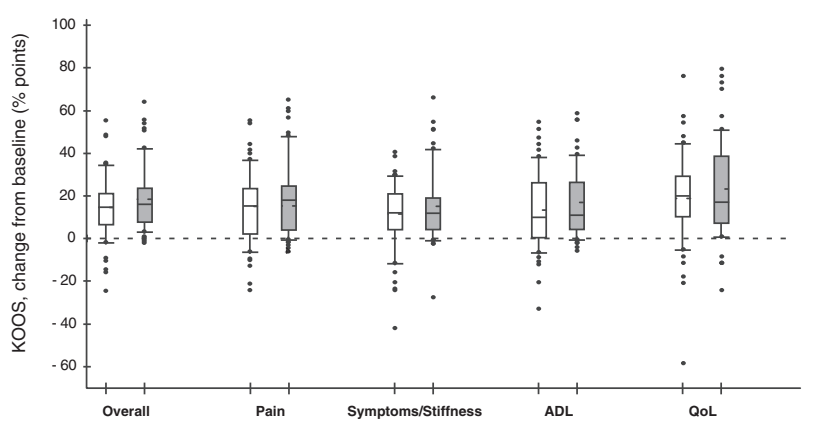

Figure 4. Change from baseline in overall KOOS and individual KOOS subdomains for the mean of 12 and 18 months of postsurgery follow-up. The clear bars represent data from the microfracture group, and the shaded bars represent data from the $\mathrm{CCl}$ group. The upper and lower edges of each box plot indicate the 25th and 75th percentiles; the "whiskers," the 10th and 90th percentiles; the solid horizontal line, the median; and the dotted line, the mean. All outliers are shown as individual data points. ADL, activities of daily living; $\mathrm{CCl}$, characterized chondrocyte implantation; KOOS, Knee injury and Osteoarthritis Outcome Score; QoL, quality of life.

groups). Approximately two thirds (38/57 [67\%]) of patients in the CCI group experienced AEs that were considered related to the study procedure, compared with 36 of $61(59 \%)$ in the MF group. Of these, 8 of 61 (13\%) in the MF group and 5 of $57(8.8 \%)$ in the CCI group were reported as serious AEs.

In both treatment groups, arthralgia was the most commonly reported $\mathrm{AE}(35 / 57$ [61\%] vs 35/61 [57\%] patients in the CCI and MF groups, respectively). Overall, significantly more patients in the CCI group experienced joint swelling (11/57 [19\%] vs 3/61 [4.9\%] patients; $P=.022)$, which occurred between 1 and 14 days after arthrotomy in the CCI group. Related AEs of joint crepitation were significantly more common in the CCI group than in the MF group (7/57 [12\%] vs $1 / 61$ [1.6\%] patients; $P=.028$ ).

Concerns have been expressed in the literature regarding cartilage hypertrophy after ACI. ${ }^{4}$ The incidence of hypertrophy, as judged by the surgeon at end point biopsy, was 14 patients $(25 \%)$ versus 8 patients $(13 \%)$ for the CCI and MF groups, respectively (no statistical difference, $P=.156$ ). Of these, 7 of 14 patients in the CCI group were symptomatic, and in the MF group, 1 of 8 was symptomatic.

Events considered related to the study procedure in the CCI group, and that required hospitalization (ie, serious AEs), included 1 case of deep vein thrombosis that occurred 19 days after surgery in a patient who had not received prophylactic heparin (the event resolved without sequelae after treatment) and 1 case of severe tendinitis of the fascia lata approximately 18 months after CCI. There were no reports of serious AEs that were considered related to the study procedure in the MF group.

No patients were discontinued from the study because of AEs, and there were no clinically important trends in the results of physical examination, vital signs, electrocardiogram, hematology studies, or clinical chemistry during the study.

\section{DISCUSSION}

This RCT is the first prospectively designed and controlled Guidelines for Good Clinical Practice-compliant trial to be conducted to date in the field of cartilage repair. This trial design included various measures to ensure an unbiased comparison, including (1) random allocation of patients to treatment group, (2) implementation of the same rehabilitation program for all patients, (3) conduct of postoperative assessments by independent evaluators, (4) assessment of structural repair by independent histopathologists blinded to the patients' treatment allocation, (5) evaluation of clinical outcome according to a patient-reported outcome measure, and (6) the use of a prespecified statistical analysis plan. Although the specialist surgical skills required for ACI make a comparison with standard surgical procedures such as MF difficult, the surgeons involved in this trial were fully trained in the CCI technique before its commencement. In addition, a coordinating surgeon was responsible for standardizing surgical procedures. Furthermore, exploratory analyses revealed no significant effects of surgeon learning on the primary end points of this study.

Both treatment groups were generally well balanced for demographic and baseline characteristics. The mean defect size treated in this study (approximately $2.5 \mathrm{~cm}^{2}$ ) is typically encountered in clinical practice and may be expected to respond well to both $\mathrm{MF}$ and $\mathrm{ACI} .^{26}$ In this study, patients in the CCI group had experienced symptoms for longer than did patients in the MF group. In addition, more patients in the CCI group had undergone knee surgery before their participation in this study. It has been shown that it is more difficult to regenerate cartilage of a hyaline-like quality when the patient has more symptoms, has had previous knee surgery, or has an older defect, ${ }^{38}$ and the randomization outcome of this study might therefore be interpreted as giving a small bias in favor of the MF group.

This study demonstrates that CCI results in superior structural repair compared with MF. Statistically significant improvements with CCI compared with MF were observed for quantitative histomorphometry, the overall histology assessment score, and certain components of structural repair relating to chondrocyte phenotype and tissue structure. Thus, analysis of the tissue regenerate at 12 months after treatment indicates that CCI is associated with more chondrocytelike cells and a higher proteoglycan content of the cellular matrix, which confers compressive strength. The repair tissue of patients treated with CCI was less fibrous and showed elements indicative of higher compressive strength and physiological maturation, in favor of true regeneration and reconstitution of articular cartilage structure.

The modified O'Driscoll score (MODS) is a widely used tool for the histologic assessment of cartilage repair in animal studies ex vivo. ${ }^{29}$ Although we initially intended to use the MODS in this study, limitations in its use in biopsy specimens of patient cartilage repair studies were reported. ${ }^{35}$ The overall histology assessment score and the individual components of cartilage repair evaluated in this study constitute a novel MODS-derived scoring system that has been designed and validated by the Histology Working Group of the International Cartilage Repair 
Society to ensure greater applicability to patient studies (P. Mainil-Varlet, unpublished data).

The basic premise of conventional ACI is that implanted chondrocytes are able to form hyaline or hyaline-like cartilage, which in turn may restore the integrity of the joint surface and promote durable tissue regeneration. It is suggested that repair tissue composed of fibrocartilage has a higher likelihood of developing morphologic anomalies and becoming symptomatic earlier than do hyaline or hyalinelike repairs. ${ }^{16}$ The only other RCT conducted to date that has compared ACI and MF failed to show a statistically significant difference between treatments in structural repair at 24 months. ${ }^{24}$ One possible explanation for the statistically significant improvement in structural repair with CCI reported for the current study, compared with the other RCT, may be related to the larger sample size (and the higher number of end point biopsies) in this study. Another possible explanation is that the characterized chondrocytes of CCI (which have been selected based on a gene marker profile predictive of their capacity to form hyaline-like cartilage) result in improved structural repair compared with the uncharacterized, dedifferentiated chondrocytes of ACI, which may have lost their ability to reexpress the articular cartilage phenotype in vivo. Indeed, a recent review of cell therapy in cardiac medicine suggested that the efficacy of cell therapy may depend on cell characterization and that cell therapy using heterogeneous populations of uncharacterized cells may also account for the disparate results in cell therapy studies in the cardiac field. ${ }^{37}$

Remodeling and maturation of the cartilage repair tissue after CCI is a process that is thought to occur over time and may continue to improve beyond 18 months after treatment. $^{25,34}$ Furthermore, the mechanisms of tissue repair with marrow stimulation techniques, such as $\mathrm{MF}$, are quite different than those with ACI, and the resulting repair tissue is thought to be more callus-like or fibrocartilaginous in nature and potentially less durable than the original articular cartilage, degenerating over time so that clinical symptoms relapse. ${ }^{5,6,21,25,27,28}$ In addition, as this method of repair is osteochondral, violating the tidemark and subchondral bone plate, it is suggested that the tidemark/bone front may move up and ultimately lead to intralesional osteophytes, which potentially contribute to the deterioration of symptoms and development of osteoarthritis. ${ }^{5}$ As a result of these 2 ongoing processes (ie, continued remodeling of regenerated tissue after ACI and degeneration of the repair tissue after $\mathrm{MF}$ ), the expected clinical benefit of CCI may not be anticipated to become manifest until at least 2 to 3 years after surgery. The results of this study confirm that CCI was not inferior to MF in short-term clinical outcome.

Patients randomized to the CCI group required both an arthroscopy with harvest biopsy and an arthrotomy, whereas those in the MF group required arthroscopy only (and no harvest biopsy). It may be expected that open surgery with CCI may be disadvantageous to the pace of physical recovery in the CCI group. However, the results from this study demonstrate that by 6 months after treatment, the patients' clinical status had improved compared with baseline to a similar extent in both groups. In addition, although it may be expected that CCI would be associated with a significantly worse tolerability profile, it was generally well tolerated and did not result in a marked increase in the incidence of AEs compared with MF. The patient retention rate was high for both treatment groups, with $90 \%$ (46/51) and 85\% (52/61) of patients treated with CCI or MF, respectively, still in the study at 18 months. Patient dropout was owing to withdrawal of consent (1/61 in the MF group), protocol violations (2/61 in the MF group), treatment failure (2/61 in the MF group and 1/51 in the CCI group), or the patient declining to enter into the extension phase $(4 / 61$ in the MF group and 4/51 in the CCI group).

The combined structural and clinical outcome measures assessed in this study are, in our view, warranted. Currently, insight into clinically relevant outcomes in these patient populations is limited. Analysis of appropriate reconstitution of tissue structure is thought to be predictive of successful long-term outcome and thus may reflect an essential aspect in the assessment of novel regenerative treatments. However, we believe that this is only acceptable if short-term clinical outcomes are not inferior to those associated with existing treatment methods and are accompanied by significant treatment effects, as observed in this study. The clinical outcome of patients enrolled in this study continues to be evaluated.

In conclusion, the use of characterized chondrocytes in autologous cartilage repair represents a new class of treatment that is associated with superior structural regeneration of cartilage tissue, compared with MF. In the short term, the risk-benefit profiles of CCI and MF appear to be similar, supporting a first-line use of this cell-based therapy with characterized cells. The better structural regeneration with CCI observed in this trial may predict better clinical outcome and merits long-term clinical evaluation.

\section{ACKNOWLEDGMENT}

We are indebted to Cosimo de Bari and Francesco dell'Accio for their critical contribution to the characterization of ChondroCelect. We also acknowledge Pierre Mainil-Varlet, $\mathrm{MD}, \mathrm{PhD}$, Institute of Pathology, University of Bern, Switzerland; and Rita Kandel, MD, Department of Laboratory Medicine and Pathobiology, University of Toronto, Canada, for reading the biopsies; and Boudewijn Vandamme, MD, PhD, Department of Morphology and Molecular Pathology, Leuven, Belgium, for preparing the biopsy specimens. The study was conducted by TiGenix NV Belgium. The invaluable contributions of the TiGenix Clinical Study Team and local study site teams are acknowledged. A system audit and auditing of study centers were performed by MSource Medical Development bvba, Kraainem, Belgium. Data management and statistical analysis were performed by International Drug Development Institute, Ottignies Louvain-la-Neuve, Belgium; additional statistical input was provided by Dennis O Chanter, DPhil, Satisfaction Statistical Consultancy Ltd, Bexhill-on-Sea, United Kingdom. RSG Pharma Consultants Ltd, Oxford, United Kingdom, and Ismar Healthcare, Lier, Belgium, were involved in the writing of the article. Micheline Wille, MD, performed an 
essential role in the final preparation, communication logistics, and article details. Dr Auw Yang is supported by the Anna Foundation and the Foundation "De Drie Lichten" in the Netherlands. Dr Saris is supported by the Netherlands Organization for Health Research and Development.

\section{REFERENCES}

1. Auw Yang KG, Saris DBF, Verbout AJ. The effect of synovial fluid from injured knee joints on in vitro chondrogenesis. Tissue Eng. 2006;12(10):1-8.

2. Bentley G, Biant LC, Carrington RW, et al. A prospective, randomised comparison of autologous chondrocyte implantation versus mosaicplasty for osteochondral defects in the knee. J Bone Joint Surg Br. 2003;85(2):223-230.

3. Brittberg M, Lindahl A, Nilsson A, Ohlsson C, Isaksson O, Peterson L. Treatment of deep cartilage defects in the knee with autologous chondrocyte transplantation. N Engl J Med. 1994;331:889-895.

4. Brittberg M, Peterson L, Sjogren-Jansson E, Tallheden T, Lindahl A. Articular cartilage engineering with autologous chondrocyte transplantation: a review of recent developments. J Bone Joint Surg Am. 2003;85(3):109-115.

5. Brown WE, Potter HG, Marx RG, Wickiewicz TL, Warren RF. Magnetic resonance imaging appearance of cartilage repair in the knee. Clin Orthop Relat Res. 2004;422:214-223.

6. Buckwalter JA, Mankin HJ, Grodzinsky AJ. Articular cartilage and osteoarthritis. Instr Course Lect. 2005;54:465-480.

7. Chen FS, Frenkel SR, Di Cesare PE. Chondrocyte transplantation and experimental treatment options for articular cartilage defects. Am J Orthop. 1997;26(6):396-406.

8. Curl W, Krome J, Gordon E, Rushing J, Smith B, Poehling G. Cartilage injuries: a review of 31,516 knee arthroscopies. Arthroscopy. 1997; 13:456-460

9. Dell'Accio F, De Bari C, Luyten FP. Microenvironment and phenotypic stability specify tissue formation by human articular cartilage-derived cells in vivo. Exp Cell Res. 2003;287(1):16-27.

10. Dell'Accio F, De Bari C, Luyten FP. Molecular markers predictive of the capacity of expanded human articular chondrocytes to form stable cartilage in vivo. Arthritis Rheum. 2001;44:1608-1619.

11. Dell'Accio F, Vanlauwe J, Bellemans J, Neys J, De Bari C, Luyten FP. Expanded phenotypically stable chondrocytes persist in the repair tissue and contribute to cartilage matrix formation and structural integration in a goat model of autologous chondrocyte implantation. $J$ Orthop Res. 2003;21:123-131.

12. Fu FH, Zurakowski D, Browne JE, et al. Autologous chondrocyte implantation versus debridement for treatment of full-thickness chondral defects of the knee: an observational cohort study with 3-year follow-up. Am J Sports Med. 2005;33(11):1658-1666.

13. Gelber A, Hochberg M, Mead L, Wang N, Wigley F, Klag M. Joint injury in young adults and risk for subsequent knee and hip osteoarthritis. Ann Intern Med. 2000;133(5):321-328.

14. Grana WA. Healing of articular cartilage: a review. Am J Knee Surg. 2000;13(1):29-32.

15. Hambly K, Bobic V, Wondrasch B, Van Assche D, Marlovits S. Autologous chondrocyte implantation postoperative care and rehabilitation: science and practice. Am J Sports Med. 2006;34:1020-1038.

16. Henderson I, Lavigne P, Valenzuela H, Oakes B. Autologous chondrocyte implantation: superior biologic properties of hyaline cartilage repairs. Clin Orthop Relat Res. 2007;455:253-261.

17. Hjelle K, Solheim E, Strand T, Muri R, Brittberg M. Articular cartilage defects in 1000 knee arthroscopies. Arthroscopy. 2002;18(7):730-734.
18. Hochberg Y. A sharper Bonferroni procedure for multiple tests of significance. Biometrika. 1988;75:800-802.

19. Horas U, Pelinkovic D, Herr G, Aigner T, Schnettler R. Autologous chondrocyte implantation and osteochondral cylinder transplantation in cartilage repair of the knee joint: a prospective, comparative trial. $J$ Bone Joint Surg Am. 2003;85:185-192.

20. Hubbard MJS. Articular debridement versus washout for degeneration of the medial femoral condyle. $J$ Bone Joint Surg $\mathrm{Br}$. 1996;78:217-219.

21. Jackson DW, Simon TM. Chondrocyte transplantation. Arthroscopy. 1996;12(6):732-738

22. Jakobsen RB, Engebretsen L, Slauterbeck JR. An analysis of the quality of cartilage repair studies. J Bone Joint Surg Am. 2005;87:2232-2239.

23. Jones DG, Peterson L. Autologous chondrocyte implantation. J Bone Joint Surg Am. 2006;88(11):2501-2520.

24. Knutsen G, Engebretsen L, Ludvigsen T, et al. Autologous chondrocyte implantation compared with microfracture in the knee: a randomized trial. J Bone Joint Surg Am. 2004;86(3):455-464.

25. Kreuz PC, Steinwachs MR, Erggelet C, et al. Results after microfracture of full-thickness chondral defects in different compartments in the knee. Osteoarthritis Cartilage. 2006;14:1119-1125.

26. Minas T, Chiu R. Autologous chondrocyte implantation. Am J Knee Surg. 2000;13(1):41-50.

27. Minas T, Nehrer S. Current concepts in the treatment of articular cartilage defects. Orthopedics. 1997;20(6):525-538.

28. Mithoefer K, Williams RJ III, Warren RF, et al. The microfracture technique for the treatment of articular cartilage lesions in the knee: a prospective cohort study. J Bone Joint Surg Am. 2005;87(9):1911-1920.

29. O'Driscoll SW, Marx RG, Beaton DE, Miura Y, Gallay SH, Fitzsimmons JS. Validation of a simple histological, histochemical cartilage scoring system. Tissue Eng. 2001;7:313-320.

30. O'Driscoll SW, Marx RG, Fitzsimmons JS, Beaton DE. Method for automated cartilage histomorphometry. Tissue Eng. 1999;5(1):13-23.

31. Peterson L, Minas T, Brittberg M, Nilsson A, Sjögren-Jansson E, Anders L. Two- to 9-year outcome after autologous chondrocyte transplantation of the knee. Clin Orthop Relat Res. 2000;374:212-234.

32. Pocock SJ, Simon R. Sequential treatment assignment with balancing for prognostic factors in the controlled clinical trial. Biometrics. 1975;31:103-115.

33. Richardson JB, Caterson B, Evans EH, Ashton BA, Roberts S. Repair of human cartilage after implantation of autologous chondrocytes. $J$ Bone Joint Surg Br. 1999;81:1064-1068.

34. Roberts S, Hollander AP, Caterson B, Menage J, Richardson JB. Matrix turnover in human cartilage repair tissue in autologous chondrocyte implantation. Arthritis Rheum. 2001;44(11):2586-2598.

35. Roberts S, McCall IW, Darby AJ, et al. Autologous chondrocyte implantation for cartilage repair: monitoring its success by magnetic resonance imaging and histology. Arthritis Res Ther. 2003;5:R60-R73.

36. Roos EM, Roos HP, Lohmander LS, Ekdahl C, Beynnon BD. Knee Injury and Osteoarthritis Outcome Score (KOOS): development of a selfadministered outcome measure. J Orthop Sports Phys Ther. 1998;28:88-96.

37. Rosenzweig A. Cardiac cell therapy: mixed results from mixed cells. N Engl J Med. 2006;355(12):1274-1277.

38. Saris DB, Dhert WJ, Verbout AJ. Joint homeostasis: the discrepancy between old and fresh defects in cartilage repair. J Bone Joint Surg Br. 2003;85(7):1067-1076.

39. Steadman JR, Rodkey WG, Rodrigo JJ. Microfracture: surgical technique and rehabilitation to treat chondral defects. Clin Orthop Relat Res. 2001;391:S362-S369.

40. Woolf A, Pfleger B. Burden of major musculoskeletal conditions. Bull World Health Organ. 2003;81(9):646-656. 